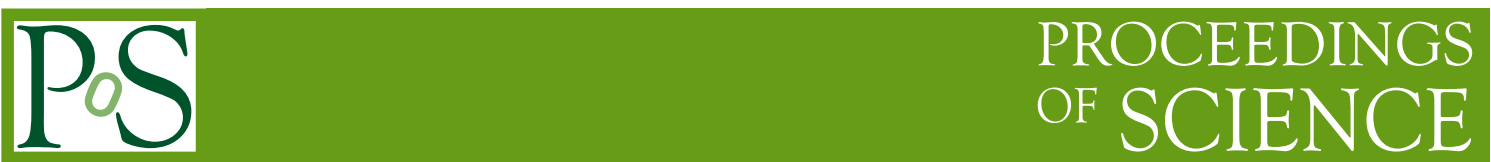

\title{
Neutron identification in the SoLid experiment
}

\author{
Simon Vercaemer ${ }^{*+}$ \\ Universiteit Antwerpen (BE), Vrije Universiteit Brussel (BE) \\ E-mail: s.vercaemer@gmail.com
}

As submitted:

The SoLid experiment aims to make a measurement of very short baseline neutrino oscillations using reactor anti-neutrinos. For this purpose, a highly segmented detector was build out of PVT cubes lined with a ${ }^{6} \mathrm{LiF}: \mathrm{ZnS}(\mathrm{Ag})$ layer.

Unlike neutrino experiments conducted deep underground, neutrino detectors used in a reactor environment need to tolerate high levels of background radiation. Therefore, a reliable distinction between the neutrons produced in inverse beta decay events and signals caused by other background interactions is crucial.

This poster presents a unique neutron identification method used in the SoLid experiment: The composite of scintillation material with different time constants enables the efficient use of pulse-shape analysis to discriminate against electromagnetic signals.

The European Physical Society Conference on High Energy Physics

22-29 July 2015

Vienna, Austria

\footnotetext{
${ }^{*}$ Speaker.

${ }^{\dagger}$ for the SoLid collaboration
} 


\section{Introduction}

The reactor anti-neutrino flux was recently recalculated for the latest generation of $\theta_{13}$ measurements [1]. Comparison of these newly calculated fluxes with measurements from the 1980s and 1990s revealed a deficit, the so-called reactor anomaly [2]. A similar deficit was measured when intense radioactive sources were used to calibrate the SAGE and GALLEX solar neutrino experiments, the Gallium anomaly [3].

Oscillations from electron to muon or tau neutrinos cannot explain these deficits, and therefore a new flavour of neutrino would be required for oscillations to explain them. Since it is known that only 3 neutrino flavours interact with the $Z$ boson[4], this new flavour cannot be allowed to interact with the $Z$ boson, hence the name 'sterile'. An oscillation from electron anti-neutrinos $\left(\bar{v}_{e}\right)$ to a sterile neutrino flavour can be used to explain both the reactor and gallium anomalies, with the best fit for possible oscillation parameters being $\sin ^{2}\left(2 \theta_{s}\right) \approx 0.1$ and $\Delta m_{s}^{2} \approx 1 e V^{2}[5]$.

The SoLid experiment aims to resolve the reactor neutrino anomaly by 2020 . This will be done by using a three-tonne, highly segmented, composite scintillator anti-neutrino detector between 5.5 and $10 \mathrm{~m}$ from the core of SCK•CEN's BR2 reactor [6]. A $288 \mathrm{~kg}$ first fully functional detector submodule (SM1) was deployed at the BR2 reactor in November 2014 [7]. SM1 successfully took reactor on, reactor off and calibration source data between February and September 2015. Data from this detector were presented in the poster.

Anti-neutrinos are detected via the process of inverse beta decay (IBD), creating a neutron and a positively charged lepton. The identification of this neutron is crucial in the reconstruction of any IBD event.

\section{Detector technology}

The SoLid detector technology is a novel detection technique designed to cope with the high background conditions found in a nuclear reactor facility. The detector is built out of $5 \times 5 \times 5 \mathrm{~cm}$ detector cubes, shown in Figure 1. Each detector cube is made from a fast scintillating cube $\left(\mathrm{PVT}^{1}, 2.1 \mathrm{~ns}\right.$ decay constant) lined with a slow scintillating material $\left({ }^{6} \mathrm{LiF}\right.$ doped $\mathrm{ZnS}: \mathrm{Ag}, 110$ ns decay constant for highly ionizing particles) and wrapped in Tyvek paper for optical isolation and improved light reflection.

The light generated in the detector

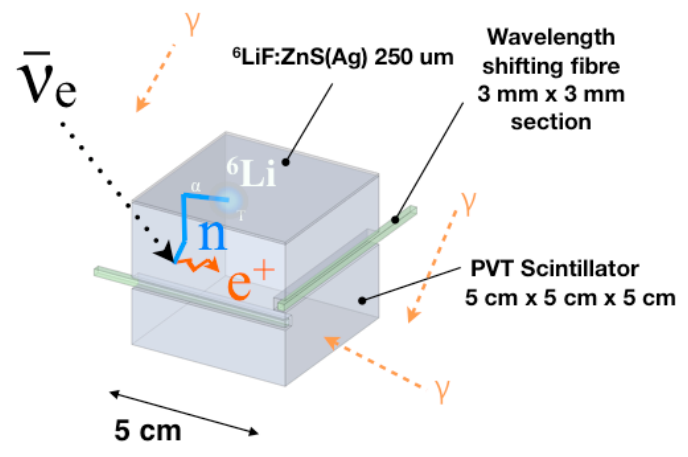
cubes is collected by two wavelength shifting fibres, one in the $\mathrm{X}$ and one in the $\mathrm{Y}$ direction, where the $\mathrm{Z}$ axis is pointing away

\footnotetext{
${ }^{1}$ Polyvinyl toluene, a plastic scintillator
} 
from the reactor core. These fibres are read out by a SiPM (Silicon Photon Multiplier) on one of the fibre ends, the other end has a mirror to reflect back the light that travelled in the opposite direction.

$\bar{v}_{e}$ interact with ${ }^{1} \mathrm{H}$ nuclei in the detector via the process of inverse beta decay (IBD, $\left.p+\bar{v}_{e} \rightarrow e^{+}+n\right)$. The positron yields a prompt scintillation pulse in PVT and two $511 \mathrm{keV}$ gammas from annihilation with an electron. Because of the high degree of segmentation, this scintillation pulse gives the IBD position with great precision, its amplitude the $\bar{v}_{e}$ energy. The neutron thermalises inside the detector and has a high probability to get captured on ${ }^{6} \mathrm{Li}$.

${ }^{6} \mathrm{Li}$ has a high thermal neutron capture cross section. After capturing a neutron, ${ }^{6} \mathrm{Li}$ splits into ${ }^{3} \mathrm{H}$ and an $\alpha$ particle, sharing $4.78 \mathrm{MeV}$ of kinetic energy. As ${ }^{3} \mathrm{H}$ and $\alpha$ are highly ionizing particles, a neutron captured on ${ }^{6} \mathrm{Li}$ induces a slowly decaying pulse in the ZnS:Ag scintillator. This stands in contrast with energy depositions in the PVT cube, which forms the bulk of the detector. Energy deposited there creates a very bright and short pulse. These two very different pulse shapes make it easy to separate signals from neutrons captured by ${ }^{6} \mathrm{Li}$ from other signals.

This combination of a prompt positron signal in PVT (called electromagnetic or EM) followed by a delayed neutron signal is a telltale sign of a $\bar{v}_{e}$ interaction in the detector. Since the detector is placed above ground and next to a nuclear reactor, there is a high background of cosmic muons and high energy gammas coming from the reactor. Because of this high EM background, it is inefficient to trigger on EM signals in general or signals that fall within the expected energy window. A trigger on a neutron capture on ${ }^{6} \mathrm{Li}$ signal is therefore desirable ${ }^{2}$. From here on, capture on ${ }^{6} \mathrm{Li}$ will be assumed for neutron signals and will not be mentioned explicitly. Neutron captures on Hydrogen are at this stage ignored.

Apart from giving a very high spatial resolution for IBD reconstruction, the segmentation also allows employing cosmic muons for energy calibration. When a cosmic muon goes through SM1, it deposits energy in a well defined set of cubes; this allows for a high precision track fit. Since the energy deposition of a minimally ionizing muon per unit distance in PVT is known, an energy calibration can be performed[8].

\section{Neutron identification}

The high decay constant for highly ionizing particles in $\mathrm{ZnS}$ :Ag is due to the high stopping power of the $\alpha$ and ${ }^{3} \mathrm{H}$ particles. When a large amount of energy is deposited over a short track length in $\mathrm{ZnS}: \mathrm{Ag}$, not only the lower lying fluorescent (short decay constant) but also the more stable excited states get populated. These excited states decay more slowly than the lower lying less stable state, giving rise to delay photons (phosphorescent light). In contrast, energy deposited in PVT is released purely fluorescent.

Recognition of these phosphorescent pulses in the waveform of a neutron capture event is key to the identification of that neutron. Multiple methods have been developed

\footnotetext{
${ }^{2}$ A neutron trigger was not in place for SM1 but is currently in development for the upgrade[7].
} 


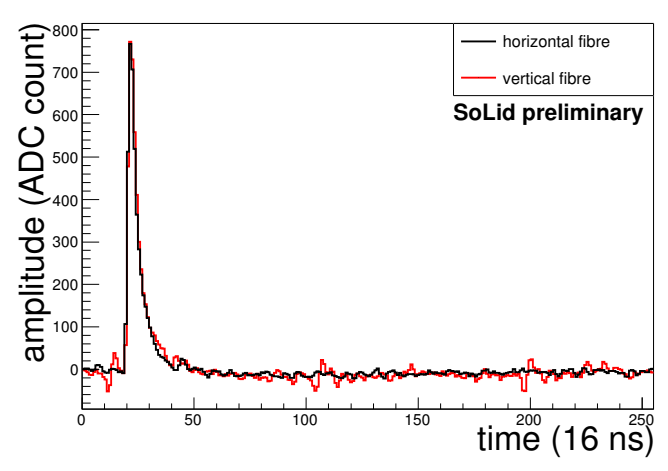

(a) An example electromagnetic waveform

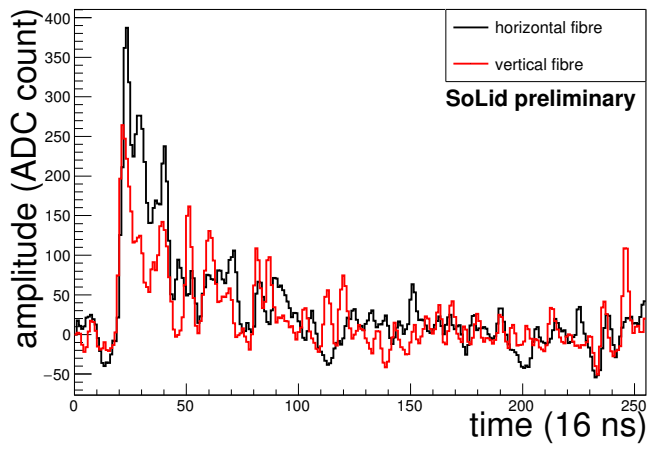

(b) An example neutron waveform

Figure 2: SM1 example waveforms

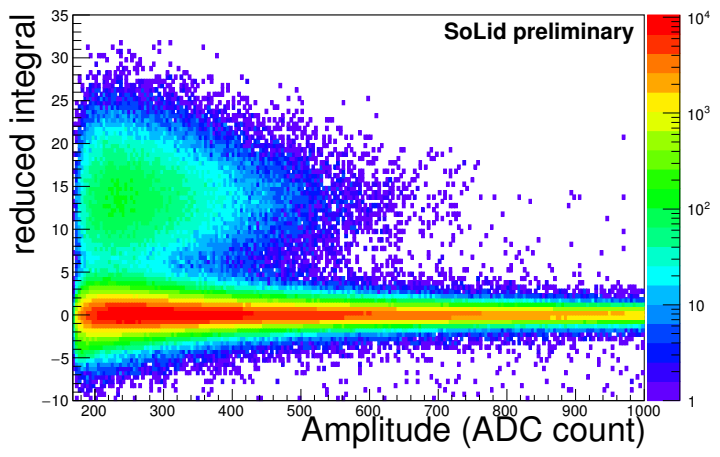

(a) SM1 AmBe data

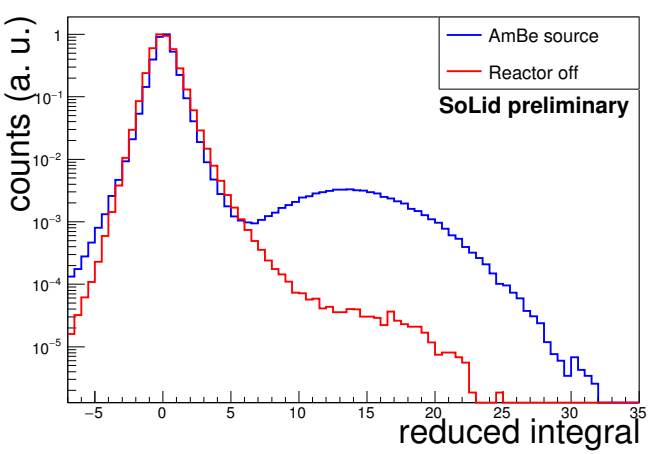

(b) AmBe to reactor off comparison

Figure 3: Averages method

to recognise this pattern, all involve the integration of the pulse. An EM pulse is purely fluorescent; its decay time is very short, as can be seen in Figure 2a. On the other hand, a waveform of a neutron captured on ${ }^{6} \mathrm{Li}$ consists of a fluorescent peak and several phosphorescent peaks later in the waveform, this can be seen in Figure 2b. Integration of such a neutron peak beyond the fluorescent peak will further increase the integral's value.

Nearly all of the energy lost by muons or positrons is deposited in one or more PVT cubes. Energy deposited in PVT is turned into photons at a rate faster than detectable by the detector's electronics. This makes the relation between the integral and the peak value of an EM signal dominated by pulse shaping effects and therefore highly linear.

\subsection{Averages method}

The simplest of the considered neutron identification methods (the averages method) uses this highly linear relationship. By calculating the integral $\left(I_{p}\right)$ and amplitude $\left(A_{p}\right)$ of a given waveform and dividing these two quantities $\left(I_{r}=I_{p} / A_{p}\right)$, it is possible to discrim- 


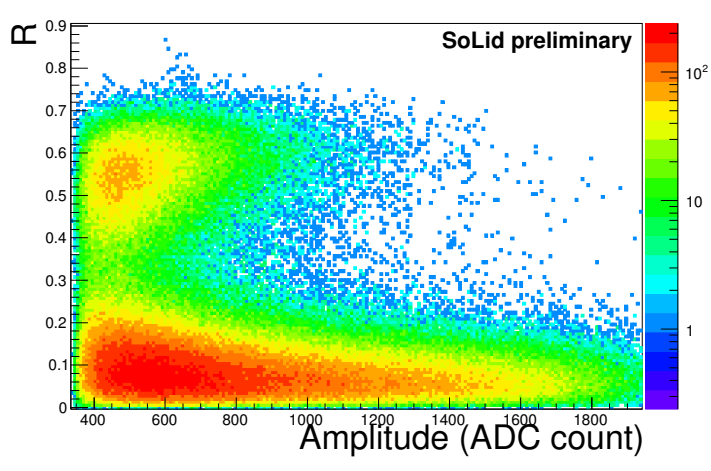

(a) SM1 AmBe data

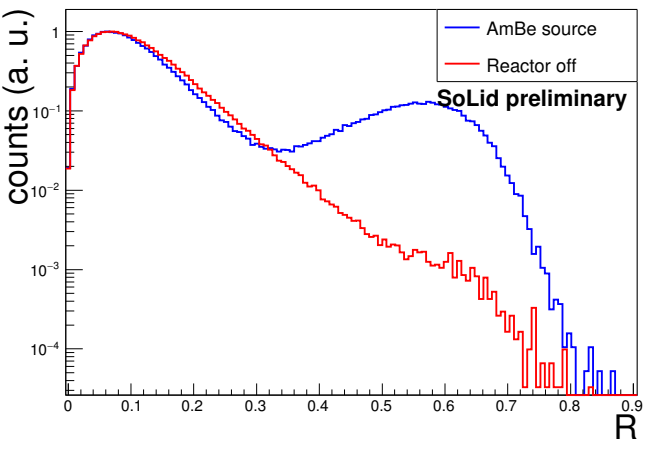

(b) AmBe to reactor off comparison

Figure 4: Charge comparison method

inate against EM signals. If the integration range is chosen such that it includes both the fluorescent and phosphorescent peaks, a different effect for EM signals and neutrons will appear. For an EM waveform, the integral value will be dominated by the first and only peak. For an neutron waveform with the same $A_{p}$ as an EM waveform, $I_{p}$ (and therefore $I_{r}$ ) will be much higher due to phosphorescent peaks.

Since for EM signals, $I_{r}$ is dominated by pulse shaping effects in the detector electronics, a channel-by-channel correction is applied. For every channel, a spectrum of $I_{r}$ is made. For a neutron rich sample, this is a spectrum with two peaks. The peak at lower values is due to EM signals, the one at higher values comes from the neutron signals. For a pure EM sample, only this lower peak will remain. As a channel correction value $\left(I_{c}\right)$, the lower peak position is chosen. Subtracting $I_{c}$ from $I_{r}$ (the 'reduced integral') takes out these channel-by-channel variations in the electronics response. This results in a spectrum centred around 0 for all channels in the detector, allowing the reduced integral to be a single cut parameter to discriminate against EM signals.

Figure 3a shows the summed reduced integral versus the summed peak amplitude for an AmBe (a neutron source) calibration run. The neutron population is clearly visible centred around a reduced integral value of 15 , the population centred around 0 are EM signals. For comparison, the reduced integral is plotted in Figure $3 \mathrm{~b}$ for an AmBe run and a reactor off run. It follows that the population at high reduced integral are neutrons since it disappears when the neutron source is taken away while all other variables were kept constant.

\subsection{Charge comparison method}

A second neutron identification method, the charge comparison method, uses a slightly more complicated discrimination method, but again uses the feature of phosphorescent light emissions. First, an integral $\left(I_{L}\right)$ is calculated such that it contains the complete fluorescent peak and a given range beyond that peak. A second integral $\left(I_{S}\right)$ that starts in the falling edge of the initial peak and runs to the same end point of $I_{L}$ is calculated. This 
integral contains part of the fluorescent and part of the phosphorescent range. For an EM signal, $I_{S}$ will have contributions only from the fluorescent part and will therefore be relatively low. For a neutron signal, contributions from phosphorescent pulses will generate a high value for $I_{S}$. Taking the ratio of $I_{S}$ and $I_{L}$ yields a value that is low for EM signals and high for neutron signals. This parameter $(\mathrm{R})$ can be used to discriminate neutron against EM signals.

In Figure $4 \mathrm{a}$, the combined $R\left(\left(I_{S x}+I_{S y}\right) /\left(I_{L x}+I_{L y}\right)\right)$ is plotted against the average peak amplitude. One can see the neutrons at high $R$ values (centred round 0.6). Here again, the comparison between an AmBe calibration run and a reactor off run is made (Figure 4b). Everything being constant except for the presence of the AmBe source, the neutron population present in the AmBe run should not be there in the reactor off run. Comparison between the two spectra shows a clear difference at high $R$ values, indicating that these are indeed neutron signals.

\subsection{Method comparison}

To qualitatively compare both methods, a figure of merit (FoM) is calculated. This FoM is defined by:

$$
F o M=\frac{\mid E M_{\text {pos }}-\text { neutron }_{\text {pos }} \mid}{\text { EM }_{\text {fwhm }}+\text { neutron }_{f w h m}}
$$

where $x_{p o s}$ is the peak position of distribution $x$ and $x_{f w h m}$ is the full width at half maximum of distribution $x$. The FoM is calculated from the AmBe run since sufficient statistics are readily available, which is not the case for reactor off data. The FoM is independent from the neutron source, the cross section for neutron capture on ${ }^{6} \mathrm{Li}$ quickly drops with increasing energy. The calculated values of FoM for average and charge comparison methods are 2.76 and 2.3 , respectively.

In this qualitative comparison, only the relative values are meaningful. A method that has a higher FoM is better since it indicates that the EM and neutron populations are more easily separated, resulting in a better trade off between purity and efficiency of the neutron sample. In the comparison of these two methods, the averages method has the better FoM ${ }^{3}$.

A quantitative comparison between the two methods was performed by having them process the same AmBe calibration sample and tag each event as either a neutron or not a neutron. Comparing all these tags results in a $99.85 \%$ agreement between the methods. When considering only the combined neutron set, all events where at least one method identified the event as a neutron, the agreement between the two methods dropped to $92.07 \%$. From the $0.15 \%$ disagreement between the two methods, $55.82 \%$ are considered a neutron by the averages method but not by the charge comparison method.

\footnotetext{
${ }^{3}$ Research into combining these two methods and further optimizing the integration ranges for the charge comparison method is ongoing.
} 


\section{Conclusion}

A new type of neutrino detector, making use of composite scintillators with different time constants, was deployed. Two methods for identifying neutrons (needed to detect inverse beta decays) were developed and some initial tests were performed. Both methods have an agreement of $99.85 \%$ on the full sample and an agreement of $92.07 \%$ in the combined neutron sample (events where at least one method tagged the event as a neutron).

\section{Acknowledgements}

The SoLid Collaboration would like to thank SCK•CEN and the BR2 reactor support staff for the very successful operation of the BR2 reactor at which the SoLid detector is located. The SoLid collaboration would like to acknowledge the funding received from the Carnot Institutes, the Flemish Hercules Foundation (Vlaamse Herculesstichting), the Research Foundation - Flanders (FWO) and the Science and Technology Facilities Council, and the University of Oxford's particle physics sub-department.

\section{References}

[1] Th. A. Mueller et al, Improved predictions of reactor antineutrino spectra, Phys. Rev. C 83 (2011), 054615.

[2] G. Mention et al, Reactor antineutrino anomaly, Phys. Rev. D 83 (2011), 073006.

[3] C. Giunti and Marco Laveder, Statistical significance of the gallium anomaly, Phys. Rev. C 83 (2011), 065504.

[4] Schael, S. et al, Precision electroweak measurements on the Z resonance, Phys. Rept. 427 (2006), 257-454.

[5] J. Kopp et al, Sterile neutrino oscillations: the global picture, JHEP 05 (2013) 050.

[6] N. Ryder et al. (The SoLid Collaboration), First results of the deployment of a SoLid detector module at the SCK•CEN BR2 reactor, these proceedings (2015).

[7] C. Moortgat et al. (The SoLid Collaboration), Construction of the SoLid reactor anti-neutrino detector, these proceedings (2015).

[8] D. Saunders et al. (The SoLid Collaboration), Muon calibration at SoLid, these proceedings (2015). 